\title{
Pitiríase rósea purpúrica: relato de caso e revisão da literatura*
}

\author{
Purpuric pityriasis rosea: case report and literature \\ review*
}

\author{
Sergio Gabriel Carbia ${ }^{1}$ \\ Myriam Chain ${ }^{2}$ \\ Ignacio Dei-Cas ${ }^{2}$ \\ Adrián Hochman ${ }^{3}$ \\ César Lagodín ${ }^{3}$ \\ Alberto Devés ${ }^{4}$ \\ Alberto Woscoff ${ }^{5}$
}

Resumo: A pitiríase rósea purpúrica constitui doença rara e 10 casos foram publicados na Europa e EUA. 0 quadro clínico cutâneo é a forma hemorrágica ou purpúrica com variável descamação marginal. Relatase 0 caso de um homem de 25 anos de idade com lesões na região escapular. A revisão da literatura enfatiza o diagnóstico diferencial das lesões purpúricas. Segundo Lilacs e Medline, não foram relatados casos na literatura latino-americana.

Palavras-chave: Exantema; pitiríase; pitiríase rósea.

Summary: Purpuric pityriasis rosea is an unusual disease with ten published cases in the American and European literature. The main feature is an hemorrhagic or purpuric eruption with or without scaling. A case is reported of a 25-year-old man with skin lesions affecting the scapular region. The revi ew emphasizes the differential diagnosis of purpuric cutaneous diseases. According to LILACS and MEDLINE, no similar case has been reported in the Latin-American literature.

Key words: Exanthema; pityriasis; pityriasis rosea.

\section{INTRODUÇÃO}

A pitiríase rósea (PR) é dermatose aguda e inflamatória de origem desconhecida. ${ }^{1}$ Casos atípicos são comuns e colocam à prova a experiência e a perspicácia clínica do médico. Entre as variantes clínicas, as lesões purpúricas raramente têm sido descritas. ${ }^{2,3,4}$ Relata-se o caso de um homem de 25 anos de idade, que apresenta PR purpúrica sem herald patch.

\section{RELATO DE CASO}

Duas semanas antes da consulta, um homem branco percebeu algumas manchas assintomáticas em seu dorso. Em seu histórico não constavam outras lesões ou sintomas

\section{INTRODUCTION}

Pityriasis rosea $(P R)$ is an acute, inflammatory der matosis of unknown origin. ${ }^{l}$ Atypical cases are not uncom mon and test the experience and clinical acumen of the physician. Among these clinical variants, purpuric lesions have rarely been described. ${ }^{2,3,4}$ We report a case of purpuric $P R$ without the "herald patch" in a 25-year-old man.

\section{CASE REPORT}

Two weeks prior to the consultation, a 25-year-old white man noticed several asymptomatic spots on his dor sum. He had no history of other skin lesions or symptoms.

\footnotetext{
Recebido em 03.01.2001. / Received in January, $03^{\text {th }}$ of 2001.

Aprovado pelo Conselho Consultivo e aceito para publicação em 14.08.2002. / Approved by the Consultive Council and accepted for publication in August, $14^{\text {st }}$ of 2002.

* Trabalho realizado na Cátedra de Dermatologia. Hospital de Clínicas José de San Martín. Escola de medicina. Universidade de Buenos Aires, Buenos Aires, Argentina. / Work done at

"Cátedra de Dermatología. Hospital de Clínicas José de San Martín". School of Medicine. University of Buenos Aires. Buenos Aires. Argentine.

Residente-chefe / Chief resident.

Residente. / Resident.

Dermatologista. / Staff dermatologist.

${ }^{4}$ Dermopatologista. Divisão de Patologia. / Dermopathologist. Patology division.

Professor titular consultor. / Titular consultant professor.

C)2003 by Anais Brasileiros de Dermatologia
} 
de pele. Ele não tinha tomado medicações.

O exame físico revelou erupções de máculas purpúricas múltiplas e simétricas sem escamação na região escapular. Eram caracterizadas por lesões ovais, com diâmetro variável de 0,5 a $1 \mathrm{~cm}$ e eixo orientado ao longo das linhas de clivagem (Figuras 1 e 2). Não foram encontradas lesões na boca.

A contagem completa das células do sangue com plaquetas, a taxa da sedimentação, o tempo de protrombina (Quick), o tempo de tromboplastina parcial (KPTT) e a análise da urina apresentaram resultados normais. Venereal Diseases Research Laboratories - laboratoriais de pesquisa de doenças venéreas - (VDRL) e os títulos dos anticorpos estreptocócicos (Asto) não foram notáveis. Outras análises de rotina, como função renal ou hepática, também foram normais.

No exame histopatológico, a lesão purpúrica revelou dermatite subaguda, com espongiose focal, infiltração celular perivascular mononuclear com extravasamento de eritrócitos na derme papilar e dilatação dos capilares. Não havia evidência de células disceratóticas na epiderme. Não foram encontrados eosinófilos e vasculites. (Figura 3)

Foi feito diagnóstico de PR purpúrica.

Nenhum tratamento farmacológico foi indicado, e sua erupção clareou no período de seis semanas a partir do início da ocorrência.

\section{DISCUSSÃO}

Apitiríase rósea $(\mathrm{PR})$ começa muitas vezes com uma placa primária escapular (herald patch) seguida de erupção papuloescamosa secundária, com distribuição característica no tronco e nas extremidades ao longo das duas próximas semanas. Trata-se de um caso clássico, e a comprovação histológica é raramente necessária. Contudo, os casos atípicos de PR chegam a cerca de $20 \%$. As lesões primárias podem ser duplas, múltiplas ou ausentes (como no caso aqui apresentado). ${ }^{1.5}$ A PR atípica pode representar diferentes variações clínicas (Tabela 1). ${ }^{15,6} \mathrm{~A} \mathrm{PR}^{2}$ purpúrica ou hemorrágica é uma

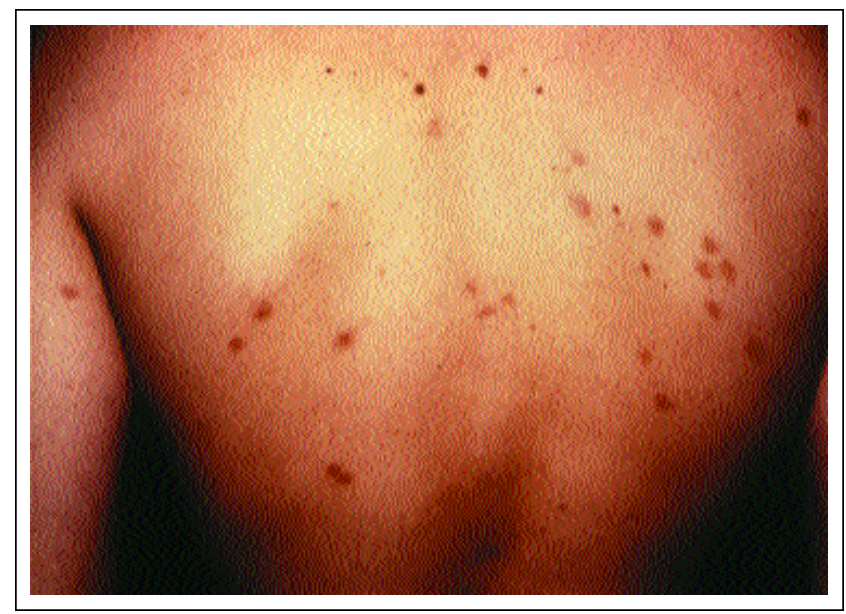

Figura 1: Erupção purpúrica com distribuição que se assemelha a padrão tipo "árvore de Natal" no dorso. / Figure 1: Purpuric eruption with distribution that resembles a "Christmas tree" pattern on the back.
He had taken no medication.

Physical examination revealed multiple, symmetri cal, non-scaling macular purpuric eruptions on the scapular region. They were characterized by oval lesions, 0.5-to$1 \mathrm{~cm}$ in diameter, with the long axis oriented along the lines of cleavage (Figures 1 and 2). No lesions were found in the mouth.

Complete blood cell count with platelets, sedi mentation rate, prothrombin time (Quick), partial throm boplastin time (KPTT) and urinalysis were normal. Venereal Diseases Research Laboratories (VDRL) and streptococcal antibody titers (ASTO) were unremarkable. Other routine analyses like renal or hepatic function were also normal.

Histopathologic examination of one purpuric lesion revealed a subacute dermatitis with focal spongiosis, peri vascular mononuclear cell infiltrates with extravasation of erythrocytes in the papillary dermis, and dilatation of capillaries. There was no evidence of dyskeratotic cells in the epidermis. Eosinophils and vasculitis were absent (Figure 3).

The diagnosis of purpuric PR was made.

No pharmacologic treatment was indicated and his eruption cleared within 6 weeks from the onset.

\section{DISCUSSION}

Pityriasis rosea $(P R)$ often starts with a primary scaly plaque ("herald patch") followed by a secondary papulosquamous eruption typically distributed on the trunk and proximal extremities two weeks later. ${ }^{1}$ This is a classic case and histologic confirmation is rarely needed. However, atypical cases of PR account for approximately 20\%. ${ }^{5.6}$ The primary lesions may be absent (as in our case), double or multiple. ${ }^{1,5}$ Atypical PR may represent different clinical variants (Table 1). ${ }^{1,5,6}$ Purpuric ${ }^{2}$ or hemorrhagic $P R^{3}$ is an atypical form affecting mainly children. ${ }^{2,7}$ It is characterized

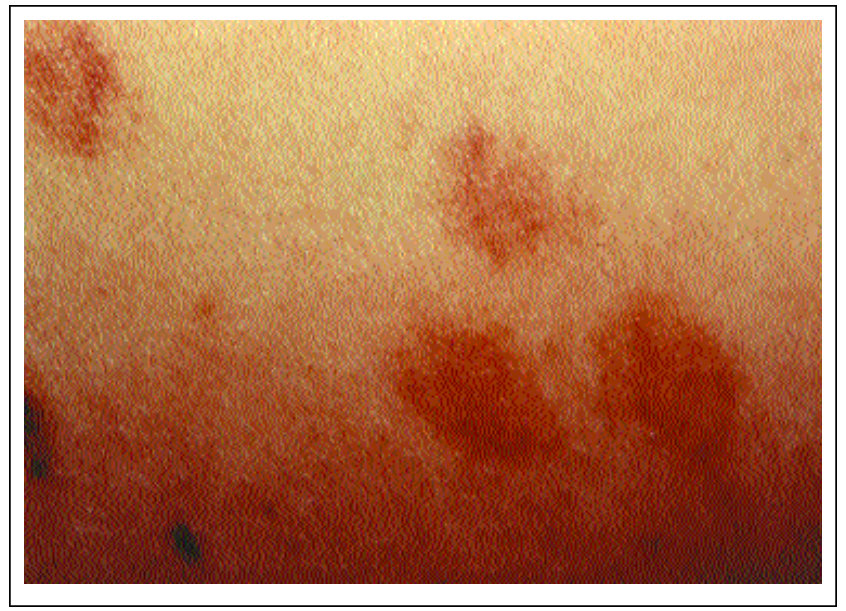

Figura 2: Lesões purpúricas em detalhe. Nota-se a ausência de escamação. / Figure 2. Purpuric lesions in detail. Note the absence of scaling. 
Figura 3: Epiderme com espongiose focal. Extravasamento de eritrócitos para dentro da derme papilar, associado com dilatação de capilares e do infiltrado perivascular mononuclear (HematoxylinaEosina, magnificação X40).

forma atípica, acometendo especialmente as crianças. ${ }^{2,7}$ É caracterizada por máculas múltiplas, pequenas e purpúricas ou hemorrágicas com ou sem escamação periférica. ${ }^{1}$

A patogênese de PR não é conhecida. ${ }^{1,6}$ As infecções causadas por estreptococos, espiroquetas, fungos e vírus têm sido implicadas em muitos estudos. ${ }^{1,5,6}$ Aprincipal evidência sugere uma etiologia viral. $\mathrm{O}$

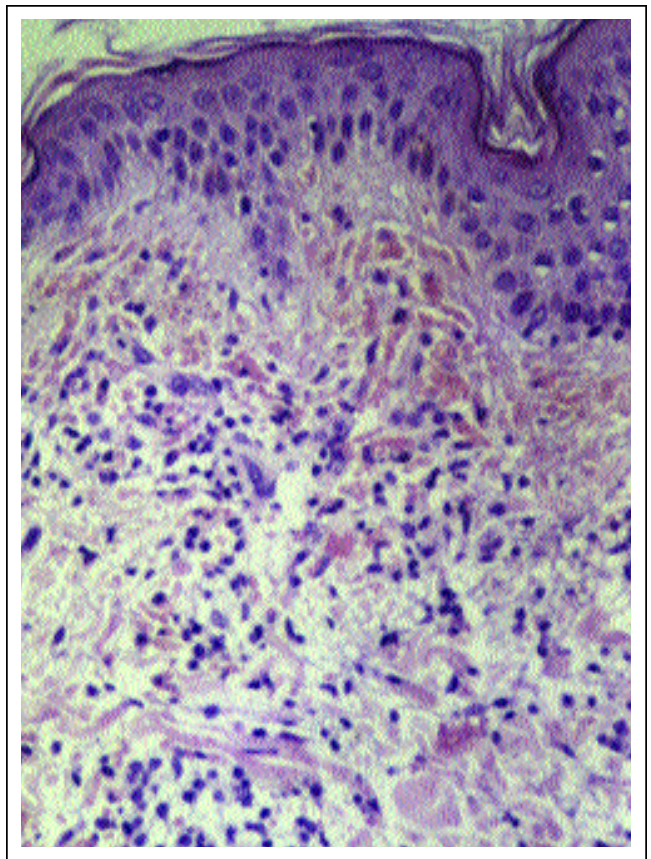

Figure 3. Epidermis with focal spon giosis. Extravasation of erythrocytes into the papillary dermis, associated with dilatation of capillaries and mononuclear perivascular infiltrate (Hematoxylin-eosin, original magnification $x 40$ ). by multiple, small, purpuric or hemorrhagic macules with or without peripheral scaling. ${ }^{1}$

The pathogenesis of $P R$ is unknown. ${ }^{1,6}$ Infections caused by streptococci, spirochetes, fungi, and viruses have been implicated in many studies. ${ }^{15,6}$ The main evi dence suggest a viral etiology. Human herpes virus type 6 and 7 (HHV-6; HHV-7), parvovirus B19, vírus herpes humano tipo 6 e 7 (VHH-6; VHH-7), o parvovírus B19 e o picornavírus foram propostos como agentes causais. Mas, apesar de os estudos ultra-estruturais terem demonstrado algumas partículas de vírus-símile na lesão primária, ${ }^{9}$ outros exames não conseguiram isolar $\mathrm{o}$ agente viral..$^{8,10,11}$ Recentemente, a principal etiologia sugerida por Drago e colaboradores $^{12}$ foi VHH-7. Contudo, esses achados não puderam ser reproduzidos nas lesões da pele, tampouco no sangue periférico ${ }^{8,13}$ As razões para a divergência não são claras.

A histopatologia da PR purpúrica é caracterizada pelo extravasamento de eritrócitos para dentro da derme papilar sem evidência de vasculite. Outros aspectos de PR típica, como paraceratose focal, espongiose e infiltrado linfócito perivascular superficial, podem estar presentes. ${ }^{2.5}$

O diagnóstico diferencial principal inclui doenças purpúricas, como vasculite, dermatoses purpúricas pigmentadas e manifestações cutâneas de doenças hematológicas (Tabela 2). ${ }^{1,45,6,14,15}$ As dermatoses purpúricas pigmentadas podem ser distinguidas porque a PR purpúrica é caracterizada pela ausência and picornavirus were proposed as causative agents. ${ }^{1,5,8}$ But despite that ultrastructural studies have demonstrated virus-like particles in the primary lesion, ${ }^{9}$ other attempts failed to isolate a viral agent..$^{8,10,11}$ Recently, the main etio logy suggested by Drago and colleagues ${ }^{12}$ was HHV-7. However, these findings could be reproduced not only in skin lesions, but also in peripheral blood. ${ }^{8,13}$ The reasons for this discrepancy are unclear.

The histopathology of purpuric PR is characterized by extravasation of erythrocytes into the papillary dermis without evidence of vasculitis. Other features of typical $P R$ such as focal parakeratosis, spongiosis, and a superficial lymphocytic perivascular infiltrate may be present. ${ }^{2,5}$

The main differential diagnosis include purpuric diseases like vasculitis, pigmented purpuric dermatoses and cutaneous manifestations of hematologic diseases (Table 2). ${ }^{1,4,5,6,14,15}$ Pigmented purpuric dermatoses can be distinguished because purpuric PR is characterized by the absence of multiple, pinpoint, red or purple macules that

Tabela 1: Variantes clínicas de PR atípica. / Table 1: Clinical variants of atypical PR.

\begin{tabular}{llll}
\hline 1.- & Papulosa / Papular & $9 .-$ & Vesicular / Vesicular \\
2.- & Urticarial / Urticarial & $10 .-$ & Eritema multiforme-like / Erythema multiforme-like \\
3.- & Eczematosa / Eczematous & $11 .-$ & Pustular / Pustular \\
4.- & Psoriasiforme / Psoriasiform & $12 .-$ & Gigantéia (incluindo circinada e marginada) \\
5.- & Liquenóide / Lichenoid & & Gigantea (circinated and marginated included) \\
6- & Purpúrica / Purpuric & $13 .-$ & Localizada / Localized \\
7.- & Pigmentada / Pigmented & 14 - & Inverso / Inverse \\
8.- & Eritrodérmica / Erythrodermic & $15 .-$ & Unilateral / Unilateral \\
\hline
\end{tabular}


Tabela 2: Diagnóstico diferencial de PR purpúrica (com ou sem escamação). Table 2: Differential diagnosis of purpuric PR (with or without scaling).

\begin{tabular}{|c|c|c|c|}
\hline A.- & $\begin{array}{l}\text { Dermatoses que induzem ou imitam lesões purpúricas } \\
\text { Dermatoses which induce or mimic purpuric lesions }\end{array}$ & B.- & $\begin{array}{l}\text { Dermatoses que induzem lesões secundárias PR-símile } \\
\text { Dermatoses which induce PR-like secondary lesions }\end{array}$ \\
\hline 1. & $\begin{array}{l}\text { Dermatoses purpúricas pigmentadas } \\
\text { Pigmented purpuric dermatoses }\end{array}$ & $\begin{array}{l}1 . \\
2 .\end{array}$ & $\begin{array}{l}\text { Tínea versicolor / Tinea versicolor } \\
\text { Eczema numular / Nummular eczema }\end{array}$ \\
\hline 2. & $\begin{array}{l}\text { Vasculites cutâneas } \\
\text { Cutaneous vasculitis }\end{array}$ & 3. & Psoriasis guttata / Guttate psoriasis \\
\hline 3. & $\begin{array}{l}\text { Sarcoma de Kaposi } \\
\text { Kaposi`s sarcoma }\end{array}$ & $\begin{array}{l}4 . \\
5 .\end{array}$ & $\begin{array}{l}\text { Sífilis secundária / Secondary syphilis } \\
\text { Líquen plano / Lichen planus }\end{array}$ \\
\hline 4. & $\begin{array}{l}\text { Micose fungóide purpúrica } \\
\text { Purpuric mycosis fungoides }\end{array}$ & 6. & $\begin{array}{l}\text { Pitiríase liquenóide crônica / Pityriasis lichenoides chronica } \\
\text { Erupções por drogas / Drug eruptions** }\end{array}$ \\
\hline 5. & $\begin{array}{l}\text { Doenças hematológicas* } \\
\text { Hematologic diseases* }\end{array}$ & 8. & $\begin{array}{l}\text { Linfomas (Hodgkin e cutâneo célula T) } \\
\text { Lymphomas (Hodgkin and cutaneous T-cell) }\end{array}$ \\
\hline 6. & $\begin{array}{l}\text { Traumatismo } \\
\text { Trauma }\end{array}$ & & $\begin{array}{l}\text { Carcinomas sólidos (estômago, pulmão) } \\
\text { Solid carcinomas (stomach, lung) }\end{array}$ \\
\hline
\end{tabular}

* Linfomas, policitemia e púrpura trombocitopênica. / Lymphomas, polycythemia and thrombocytopenic purpura.

** Barbitúricos, captopril, clonidine, isotretinoína, metronidazola, ouro e penicilamina. / Barbiturates, captopril, clonidine,

isotretinoin, metronidazole, gold and penicillamine.

de múltiplas máculas minúsculas vermelhas ou arroxeadas que se assemelham a manchas de pimenta cayenne. ${ }^{6.14}$ Análise bioquímica normal (especialmente hemograma com contagem de plaquetas e estudos de coagulação) descartou doenças hematológicas, como linfomas, policitemia e purpúra trombocitopênica. As doenças mais importantes que podem imitar uma erupção secundária de PR atípica são a sífilis e as erupções PR-símile induzidas por drogas. ${ }^{1.5}$ Por isso o teste sorológico para sífilis e uma interrogação completa sobre reações às drogas (em particular barbitúricos, captopril, clonidine, isotretinoína, metronidazola, ouro e penicilamina) devem ser feitos em todos os casos atípicos. ${ }^{5}{ }^{15}$ Abiópsia da pele é necessária, visto que as vasculites, o sarcoma de Kaposi, micoses fungóides purpúricas, psoriasis guttata e líquen plano podem imitar essa doença. A pitiríase liquenóide crônica pode ser associada com uma erupção em "padrão tipo árvore de Natal", mas as lesões típicas são distintas (pápulas eritematosas múltiplas com uma escada micácea facilmente destacada por curetagem). ${ }^{1}$

A resolução espontânea da PR purpúrica em período que varia de quatro a seis semanas é a regra, sem alteração no prognóstico. ${ }^{2}$ Hartman foi o primeiro a descrever essa variante rara de PR, ${ }^{16} \mathrm{e}$ desde então apenas 10 casos foram relatados na literatura americana e européia, segundo Pierson. ${ }^{2,3,4,16,17}$ A pesquisa bibliográfica foi efetuada em Lilacs e Medline (1968-2001), e não se encontrou nenhum caso na literatura latino-americana. resemble cayenne pepper spots. ${ }^{6,14}$ Normal biochemical analysis (specially hemogram with platelet count and coagulation studies) discharged hematologic diseases such as lymphomas, polycythemia and thrombocytopenic purpura. The most important diseases that can mimic secondary eruption of atypical $P R$ are secondary syphilis and drug-induced PR-like eruptions. ${ }^{1,5}$ For that reason, serologic test for syphillis and a complete interrogation about reactions to drugs (particulary barbiturates, cap topril, clonidine, isotretinoin, metronidazole, gold and penicillamine) must be done for all atypical cases. ${ }^{5,15}$ Skin biopsy specimen is necessary because vasculitis, Kaposi's sarcoma, purpuric mycosis fungoides, guttate psoriasis and lichen planus can mimic this disease. Pityriasis lichenoides chronica can be associated with a "Christmas-tree pattern" eruption, but the typical lesions are distinctive (multiple erythematous papules with a micaceous scale easily detached by curettage). ${ }^{l}$

Spontaneous resolution of purpuric PR in 4-6 weeks is the rule, with no alteration in prognosis. ${ }^{2}$ Hartman first described this unusual variant of $P R^{16}$ and since then, there have only been 10 cases reported in the American and European literature according to Pierson. ${ }^{2,3,4,16,17}$ We con ducted the bibliographic search in LILACS and MEDLINE (1968-2001), and were not able to find any case in the Latin-American literature. 


\section{AGRADECIMENTOS}

Os autores agradecem as úteis orientações oferecidas pelas Dras. Julieta Ruiz Beguerie e Veronica Malah.

\section{REFERÊNCIAS / REFERENCES}

1. Björnberg A. Pitiriasis rosada. In: Fitzpatrick TB, Eisen AZ, Wolff K, Freedberg IM, Austen KF, eds. Dermatología en medicina general. Buenos Aires: Panamericana, 1997:1171-8.

2.Vervov J. Purpuric pityriasis rosea. Dermatologica 1980;160:420:142-5.

3. Paller AS, Esterly NB, Lucky AW, et al. Hemorrhagic pityriasis rosea: an unusual variant. Pediatrics 1982;70:357-9.

4. Pierson JC, Dijkstra JW, Elston DM. Purpuric pityriasis rosea. J Am Acad Dermatol 1993;28:1021.

5. Gonzalez E. Pityriasis rosea. In: Arndt KA, LeBoit PE, Robinson JK, Wintroub BU, eds. Cutaneous medicine and surgery. Philadelphia: WB Saunders:, 1996; 218-21.

6. de Souza Filho JJ, Filho RMA, Duarte F, Miot HA. Pitiríase rósea: uma revisão. An Bras Dermatol 1998; 73: 245-50.

7. Bari M, Cohen BA. Purpuric vesicular eruption in a 7-year-old girl: vesicular pityriasis rosea. Arch Dermatol 1990;126:1497,1500. 8. Kempf W, Adams V, Kleinhans M, et al. Pityriasis rosea is not associated with human herpesvirus 7. Arch Dermatol 1999;135:1070-2.

9. Aoshima T, Komura J, Ofuji S. Virus-like particles in the herald patch of pityriasis rosea (letter). Dermatologica 1981;162:64-5.

10. Marcus-Farber BS, Bergman R, Ben Porath E, Zaltzman N, Friedman Birnbaum R. Serum antibodies to parvovirus B19 in patients with pityriasis rosea. Dermatology 1997;194:371.

11. Aractingi S, Morinet F, Mokni M, et al. Absence of picor-

\section{ACKNOWLEDGMENTS}

The authors appreciate the helpful advice of Julieta Ruiz Beguerie M.D. and Veronica Malah M.D.

navirus genome in pityriasis rosea. Arch Dermatol Res 1996;289:60-1.

12. Drago F, Ranieri E, Malaguti F, Losi E, Rebora A. Human herpesvirus 7 in patients with pityriasis rosea. Dermatology 1997; 195:374-8.

13. Yasukawa M, Sada E, Machino H, Fujita S. Reactivation of human herpesvirus 6 in pityriasis rosea (letter). Br J Dermatol 1999;140:169-70.

14. Bowers KE. Pigmented purpuric dermatoses. In: Arndt KA, LeBoit PE, Robinson JK, Wintroub BU, eds. Cutaneous medicine and surgery. Philadelphia: WB Saunders, 1996;291-4.

15. Parsons JM. Pityriasis rosea update: 1986. J Am Acad Dermatol 1986;15:159-67.

16. Hartman MS. Pityriasis rosea. Arch Dermatol 1944;50:201. 17. Bunch LW, Tilley JC. Pityriasis rosea. A histologic and serologic study. Arch Dermatol 1961;84:79-86.

ENDEREÇO PARA CORRESPONDÊNCIA: / MAILING ADDRESS: Sergio Gabriel Carbia

Aráoz 1083 2ํA

Buenos Aires (1414) Argentina

Tel/Fax: (541) 4777-6537

E-mail: scarbia@intramed.net.ar 